\title{
CDC25A targeting by miR-483-3p decreases CCND-CDK4/6 assembly and contributes to cell cycle arrest
}

\author{
T Bertero ${ }^{1,2}$, C Gastaldi ${ }^{1,2}$, I Bourget-Ponzio ${ }^{2,3}$, B Mari ${ }^{1,2}$, G Meneguzzi ${ }^{2,3}$, P Barbry ${ }^{\star, 1,2}$, G Ponzio $^{1,2}$ and R Rezzonico ${ }^{\star, 1,2}$
}

Disruption of contact inhibition and serum afflux that occur after a tissue injury activate cell cycle, which then stops when confluence is reached again. Although the events involved in cell cycle entry have been widely documented, those managing cell cycle exit have remained so far ill defined. We have identified that the final stage of wound closure is preceded in keratinocytes by a strong accumulation of miR-483-3p, which acts as a mandatory signal triggering cell cycle arrest when confluence is reached. Blocking miR-483-3p accumulation strongly delays cell cycle exit, maintains cells into a proliferative state and retards their differentiation program. Using two models of cell cycle synchronization (i.e. mechanical injury and serum addition), we show that an ectopic upregulation of miR-483-3p blocks cell cycle progression in early G1 phase. This arrest results from a direct targeting of the CDC25A phosphatase by miR-483-3p, which can be impeded using an anti-miRNA against miR-483-3p or a protector that blocks the complex formation between miR-483-3p and the $3^{\prime}$-untranslated region (UTR) of CDC25A transcript. We show that the miRNA-induced silencing of CDC25A increases the tyrosine phosphorylation status of CDK4/6 cyclin-dependent kinases which, in turn, abolishes CDK4/6 capacity to associate with D-type cyclins. This prevents CDK4/6 kinases' activation, impairs downstream events such as cyclin E stimulation and sequesters cells in early G1. We propose this new regulatory process of cyclin-CDK association as a general mechanism coupling miRNA-mediated CDC25A invalidation to CDK posttranscriptional modifications and cell cycle control.

Cell Death and Differentiation (2013) 20, 800-811; doi:10.1038/cdd.2013.5; published online 22 February 2013

Tissue homeostasis requires precise control of cell proliferation in response to environmental cues. Progression through the G1 phase represents a rate-limiting step of any mammalian cell proliferation. Before the passage of the restriction point, activation of CCND- and CCNE-dependent CDKs initiates a sequence of events that eventually leads to the initiation of a full cell cycle. Any defective coordination of the undergoing molecular processes can lead to aberrant tissue reparation, and even tumorigenesis. ${ }^{1}$ Early induction of CCND1 by mitogens represents a critical step for initiating cell cycle progression from quiescence. Under favorable growth conditions, CDK4/6 associate with CCNDs, phosphorylate and inactivate pRB, allowing the release of E2F and the transcription of its target genes, such as cyclins $E$ and $A$. The activity of CDKs can be blocked by natural inhibitors, CKI, belonging either to the INK4 ( $16^{\text {INK4a }}, p 15^{\text {INK4b }}, p 18^{\text {INK4c }}$, $\left.\mathrm{p} 19^{\mathrm{INK} 4 \mathrm{~d}}\right)$ or the $\mathrm{CIP}\left(\mathrm{p} 21^{\mathrm{CIP} 1}, \mathrm{p} 27^{\mathrm{KIP} 1}, \mathrm{p} 57^{\mathrm{KIP} 2}\right)$ families. ${ }^{2}$

MiRNAs are small non-coding RNAs that post-transcriptionally repress gene expression, mainly through binding to the $3^{\prime}$ (untranslated regions) UTRs of their target mRNAs. It has now been well documented that regulation by miRNAs contributes significantly to the fine-tuning of cell cycle. ${ }^{3}$ Anti-proliferative miRNAs directly target CDKs (miR-16 family, miR-24, miR-33,
miR-449a/b/c, miR-125b and so on), cyclins (miR-15, miR-26a, miR-34a, miR-19a, let-7 and so on) or E2F family members (miR-1, miR-17 92, miR-21, miR-24, miR-125a, miR-210, miR-330/331), whereas pro-proliferative miRNAs have effects on the pocket protein pRB (miR-17 92, miR-106a, miR-290, miR-335) or on inhibitors of the INK4 and CIP families (miR17 92, miR-24, miR-31, miR-106b, miR-181, miR-221/222). ${ }^{3}$

CDC25A, a dual-specificity phosphatase that activates CDK complexes through dephosphorylation of tyrosine/threonine residues on CDKs, is an important actor of cell cycle. Interestingly, it is targeted by several miRNAs (miR-15a, miR16, miR-21, miR-125b, miR-424, miR-449a/b, miR-503, let-7), probably in line with their own anti-proliferative effects. ${ }^{4-12}$ The molecular pathways associating an invalidation of CDC25A to cell-growth arrest have remained so far incompletely understood.

Using in vitro and in vivo models, we recently demonstrated the accumulation of miR-483-3p during wound closure of keratinocytes and found that kinase MK2, cell proliferation marker MKI67 and transcription factor YAP1 were direct targets of miR-483-3p that could contribute to the control of keratinocyte proliferation at the final steps of re-epithelialization. ${ }^{13}$ Additional miR-483-3p target genes including $B B C 3 /$ PUMA, SMAD4, CTNNB1 and GDF3 have been identified in

${ }^{1}$ CNRS UMR 7275, IPMC, Physiological Genomics of the Eukaryotes, Valbonne, France; ${ }^{2}$ Université de Nice Sophia-Antipolis, Nice, France and ${ }^{3}$ IRCAN, Faculté de Médecine, Nice, France

${ }^{*}$ Corresponding author: R Rezzonico or P Barbry, CNRS UMR 7275, IPMC, Physiological Genomics of the Eukaryotes, 660 route des Lucioles, Valbonne F-06560, France. E-mail: rezzonico@ipmc.cnrs.fr or direction@ipmc.cnrs.fr

Keywords: CDC25A; cell cycle; keratinocyte; microRNA; wound healing

Abbreviations: CDK, cyclin-dependent kinase; CKI, CDK inhibitor; FCS, fetal calf serum; KRT, keratin; LNA, locked nucleic acid; LOI, loss of imprinting; miRNA, microRNA; mRNA, messenger RNA; NHK, Normal human keratinocytes; NICD, Notch intracellular domain; siRNA, short interfering RNA; UTR, untranslated region Received 12.10.12; revised 18.12.12; accepted 03.1.13; Edited by RA Knight; published online 22.2.13 
other tissues (Wilm's tumors, adrenocortical carcinomas, HCT116 colon and HepG2 hepatocellular carcinoma cell lines, and adipose tissue). ${ }^{14-18}$

We demonstrate now that CDC25A has a key role in the mediation of the anti-proliferative effects of miR-483-3p in keratinocytes by controlling the binding between CDK6/4 and CCNDs. Our results obtained on CDK6 shed new light on the mechanisms that control CCND-CDK6/4 assembly in early G1, showing that this association greatly depends on the phosphorylation status of the $Y$ residues located in the 'Glycin rich domain' of these kinases.

\section{Results}

MiR-483-3p blocks injured-keratinocytes in early G1. To characterize the molecular mechanism whereby miR-483-3p exerts its anti-proliferative effect, we analyzed the impact of its overexpression on cell cycle commitment stimulated by scratch wounding. When confluent NHK were submitted to injury, they entered synchronously in the cell cycle, completed a round of mitosis and were arrested in the next G1 because of renewed confluence. ${ }^{19,20}$

NHK were transiently transfected with pre-miR-483-3p or control pre-miR-NC grown to confluence and scratch wounded. Twenty-four hours after injury, about $30 \%$ of the miR-NC-treated NHK had entered in S/G2/M phases (Figure 1a), CCND1 and CCNA were stimulated, and pRB was phosphorylated on $S 780$ residue (Figure $1 \mathrm{~b}$ ). In contrast, miR-483-3p-treated NHK were blocked in G1, with CCND1 induction but without stimulation of CCNA expression and $\mathrm{pRB}$ phosphorylation, indicating that cells entered in the cell cycle but did not reach the $S$ phase. In addition, $\mathrm{p} 27^{\mathrm{KIP} 1}$ expression was significantly higher in miR-483-3ptransfected than in control keratinocytes. Similar results were observed in HaCaT-immortalized keratinocytes (Supplementary Figure S1). a
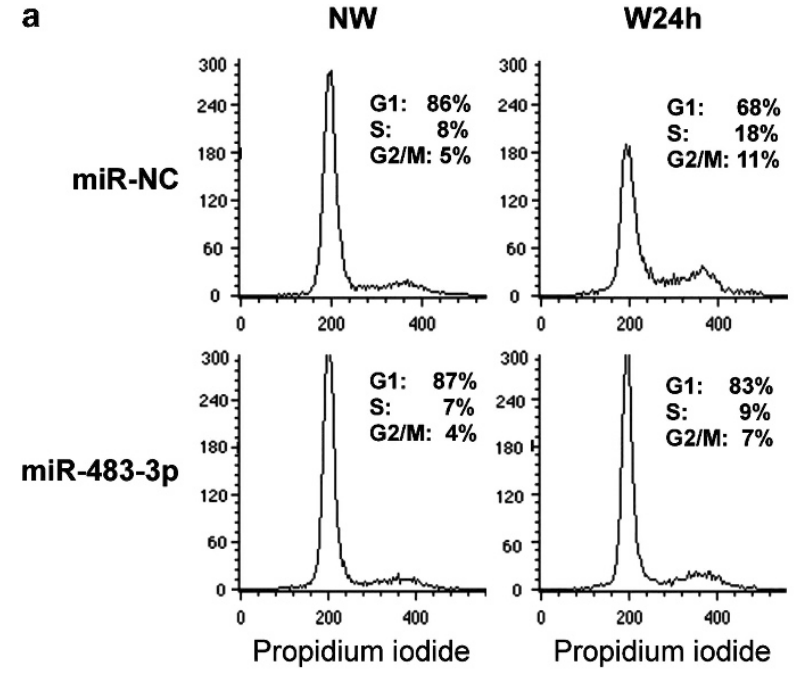

b

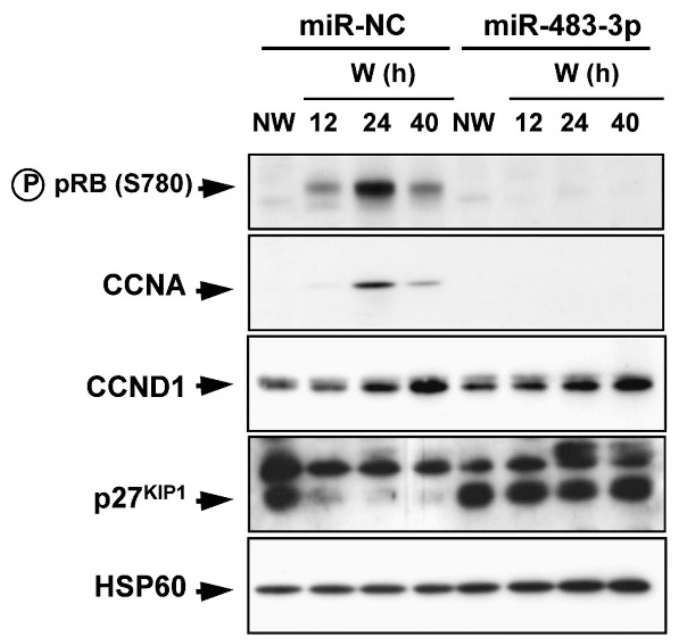

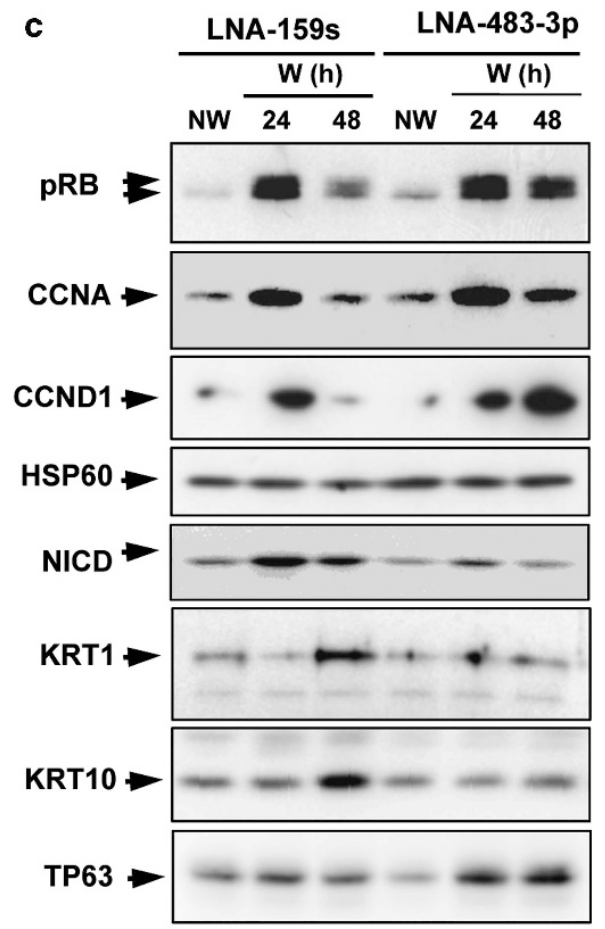

Figure 1 miR-483-3p blocks injured keratinocytes in early G1. (a) Cell cycle analysis of NHK transfected with pre-miR-483-3p or control pre-miR-NC, synchronized in G0/G1 by confluence (NW) and launched into the cell cycle for $24 \mathrm{~h}$ by scratch wounding. (b) Western blot analysis of NHK treated as in (a). (c) NHK transfected with LNA antimiR-483-3p or control LNA-159s were grown to confluence (NW) then submitted to injury and harvested after 24 and $48 \mathrm{~h}$. Expression of cell cycle and differentiation markers were analyzed by western blot. HSP60 was used as a loading control. Data are from a typical experiment chosen among five 
Interestingly, when the endogeneous stimulation of miR-483-3p by injury ${ }^{13}$ was prevented using an antagonist (LNA-483-3p), the levels of CCNA, CCND1, as well as pRB phosphorylation remained elevated $48 \mathrm{~h}$ after injury, whereas strongly decreased in control cells (LNA-159s) (Figure 1c). This observation indicates that the inactivation of miR-483-3p prevented the cell cycle exit at the end of the healing process and allowed NHK to progress into the next G1/S phase. Blocking of miR-483-3p action also precluded the activation of Notch proteolytic processing (NICD), KRT1 and KRT10 differentiation markers, and maintained TP63 expression.

MiR-483-3p inhibits the cell cycle upstream of CCNE induction. We then studied the effect of miR-483-3p on serum-induced cell cycle commitment, an experimental condition that launches more HaCaT cells into the cell cycle than scratch wounding. Pre-miR-NC and pre-miR-483-3ptransfected $\mathrm{HaCaT}$ cells were synchronized in G0/G1 by serum starvation, and released from quiescence by fetal calf serum (FCS) addition (Figure 2). HaCaT cells transfected with $\mathrm{miR}-483-3 p$ did not reach the $S$ phase upon FCS addition (15 versus $44 \%$ in control in $S$ phase after $24 \mathrm{~h}$ ) (Figure 2a). CCNE and CCNA, as well as phosphorylation of pRB, were not stimulated. Overexpression of miR-483-3p did not prevent the stimulation of CCND1 and CCND3
(Figure $2 b$ ). The analysis of the CKIs revealed that miR-483$3 p$ did not alter the expression $\mathrm{p} 15^{\mathrm{INK} 4 \mathrm{~B}}$ and $\mathrm{p} 21^{\mathrm{CIP} 1}$, but increased the level of $\mathrm{p} 27^{\mathrm{KIP} 1}$ (Figures $1 \mathrm{~b}$ and $2 \mathrm{~b}$ ). However, this upregulation was not involved in the anti-proliferative effect of miR-483-3p (Supplementary Figure S2).

The ability of miR-483-3p to block CCNE induction strongly suggests that cells were blocked in early G1 phase via a CCNE transcriptional inhibition (Supplementary Figure S3).

MiR-483-3p prevents the activation of CCND-CDK4/6 complexes. The transcriptional stimulation of CCNE is a direct consequence of the phosphorylation of $\mathrm{pRB}$ by the CCND-dependent kinases, CDK4 and CDK6. The active CCND-CDK4/6 complexes can phosphorylate pRB on specific sites, including S780, which is not phosphorylated by CCNA/E-CDK2 complexes. ${ }^{21,22}$ Consequently, the analysis of pRB (S780) phosphorylation represents a direct measurement of CDK4/6 activities. Consistent with an inhibition of the activity of CCND-CDK4/6 complexes, miR483-3p markedly decreased the phosphorylation of $p R B$ on $\mathrm{S} 780$ in FCS-stimulated HaCaT cells (Figure $2 \mathrm{~b}$ ) and in wounded NHK (Figure 1b). As this inhibition cannot be explained by any alteration in CCND1 or CCND3 expression, these observations suggest the existence of other(s) inhibitory mechanism(s). a

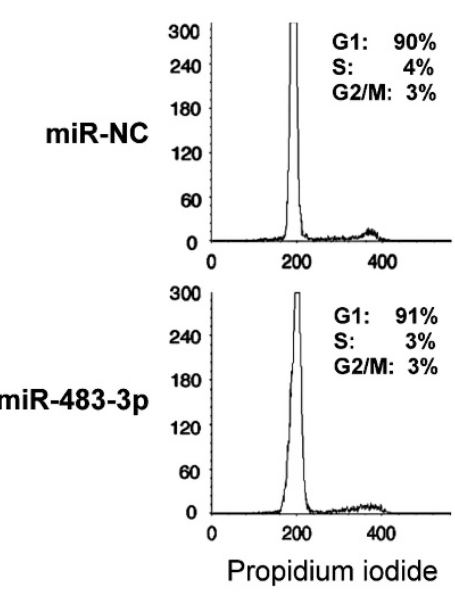

+ FCS 24h
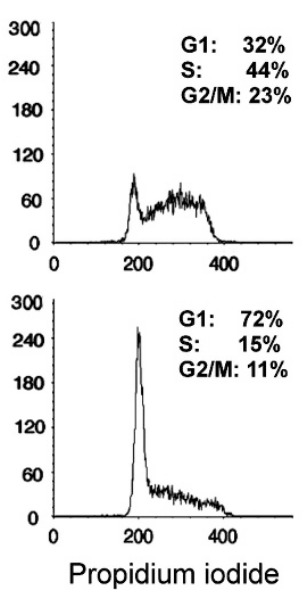

b

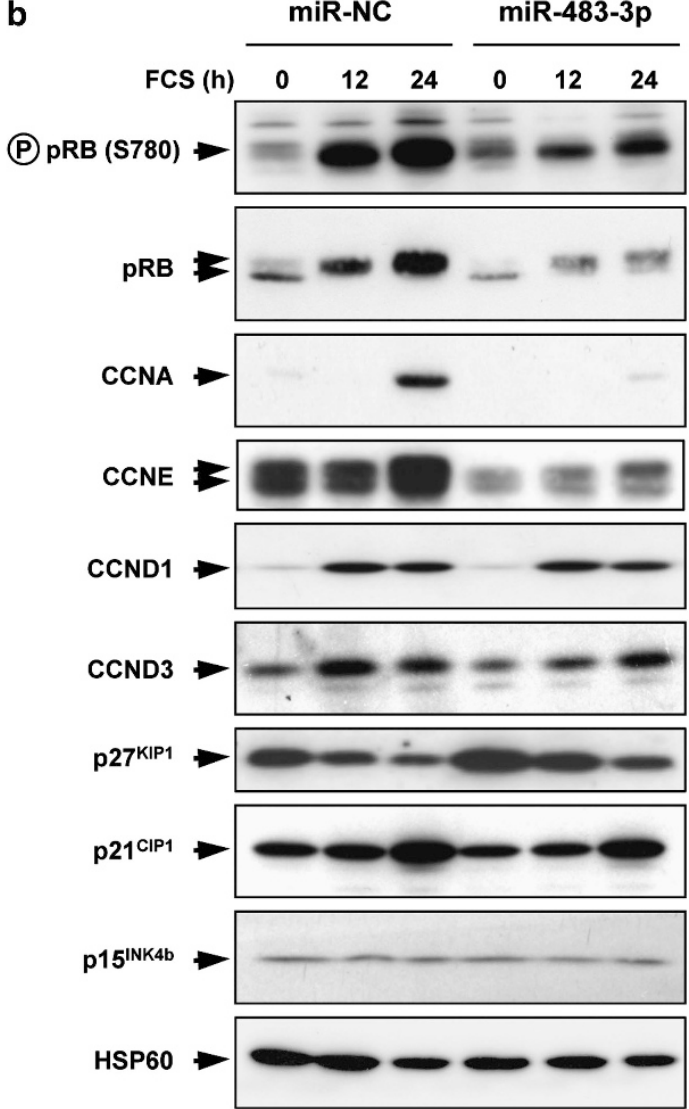

Figure 2 miR-483-3p blocks CCNE induction in synchronized HaCaT keratinocytes. (a) Cell cycle analysis of HaCaT cells transfected with pre-miR-483-3p or control premiR-NC synchronized in G0/G1 by FCS starvation and launched into the cell cycle for 12 or $24 \mathrm{~h}$ by FCS addition. (b) Western blot analysis of HaCaT cells treated as in (a). Data are from a typical experiment chosen among four 
CDK4 and CDC25A are direct targets of miR-483-3p. We looked for miR-483-3p putative targets that could interfere with CDK4/6 activity (Supplementary Table S1). We focused our attention on CDC25A, CDK4 and CDK6, as they contain one-three miR-483-3p putative binding sites in their $3^{\prime}$-UTR.

In a luciferase gene reporter assay, miR-483-3p markedly decreased luciferase activity of the CDK4 and CDC25A constructs in a range of $30-55 \%$ (Figure $3 b$ ). Surprisingly, we were unable to validate CDK6 as a direct target of miR-483-
$3 p$, despite the presence of three putative miR-483-3p sites in the $3^{\prime}$-UTR of CDK6 (Figure 3b).

The inhibitory effect of miR-483-3p was abolished after directed mutagenesis of the CDC25A and CDK4 'seed' binding sequences (Figure $3 b$ ). Both genes are thus direct targets of miR-483-3p.

Protein expression of CDC25A and CDK4 was strongly reduced in NHK and $\mathrm{HaCaT}$ cells transfected with miR-483-3p (Figure 3c). Downregulation of CDK4 and CDC25A, observed a

a CDC25A binding site1

3'-UUCUGCCCUCCUCU---CCUCACU-5'

CDC25A binding site2
5'-TTTTAGAGAC------AgGAGTGATGAAT-3'
$: 1111 \quad 111111111$
3'-UUCUGCCCUCCUCUंCCUCACU-5'

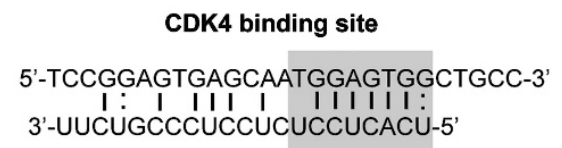

b
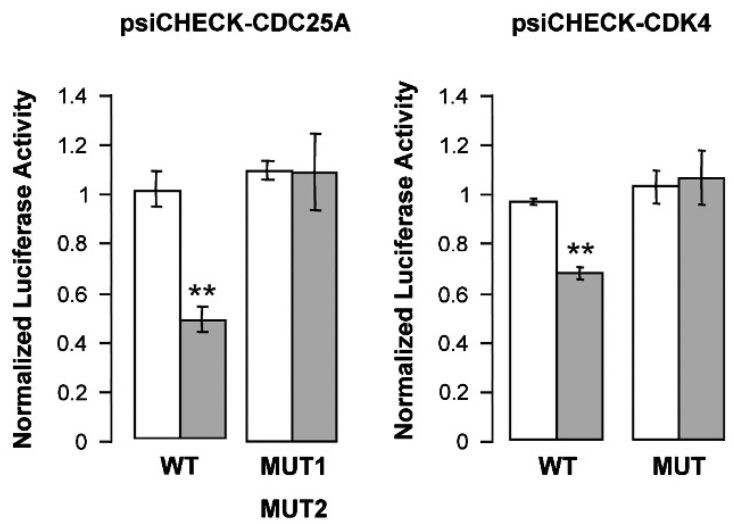

C
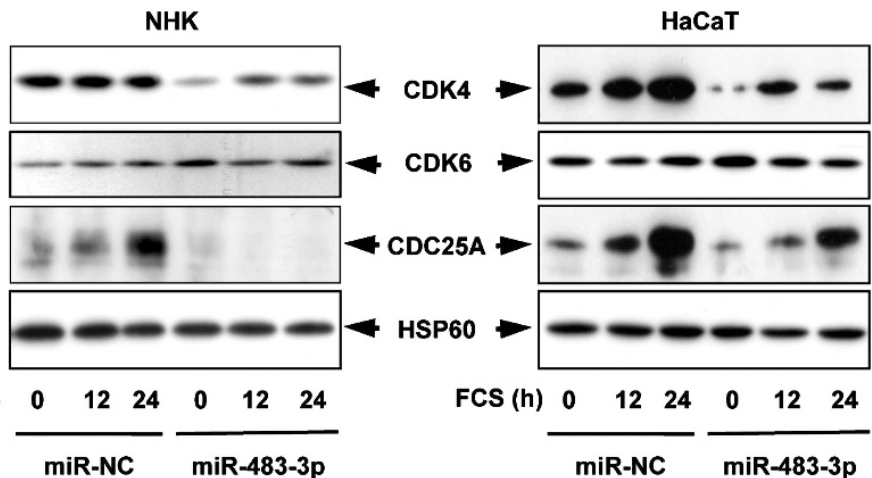
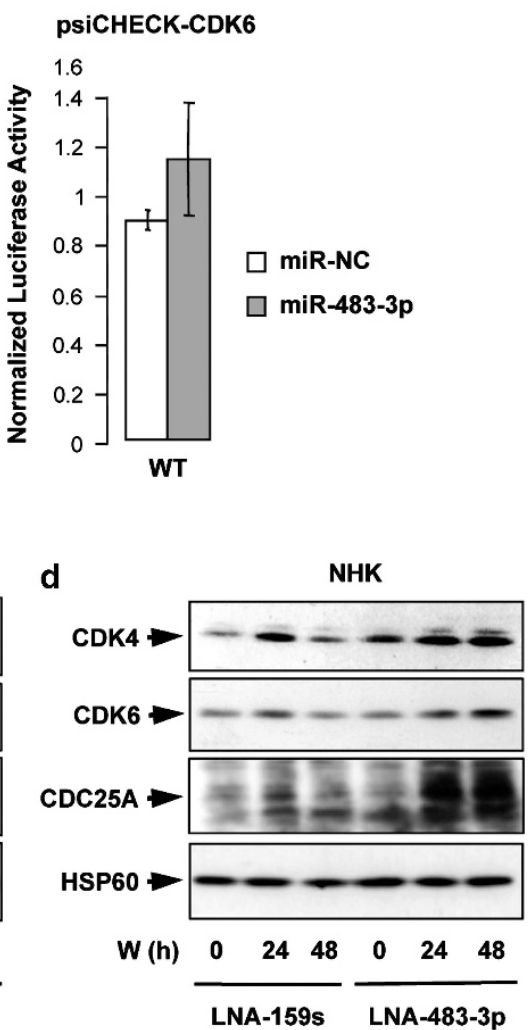

Figure 3 Identification and validation of miR-483-3p target genes. (a) Sequence of putative miR-483-3p binding sites in the $3^{\prime}-$ UTR of human CDC25A and CDK4. (b) Luciferase reporter assays of wild type (WT) and mutated (MUT) $3^{\prime}$-UTR sequences of CDC25A, CDK4 and CDK6 in the presence of pre-miR-NC or pre-miR-483-3p. Data are means \pm S.D. of normalized luciferase activity from three independent experiments. Statistically significant differences are indicated $\left({ }^{* *} P<0.001\right)$. (c) Western blot analysis of CDK4, CDK6 and CDC25A expression in NHK and HaCaT cells transfected with pre-miR-483-3p or control pre-miR-NC, synchronized in G0/G1 by confluence or FCS starvation and launched into the cell cycle for 12 or $24 \mathrm{~h}$ by scratch wounding or FCS addition, respectively. (d) NHK transfected with LNA anti-miR-483-3p or control LNA159 s were grown to confluence (NW), then submitted to injury and harvested after 24 and $48 \mathrm{~h}$. Expression of CDK4, CDK6 and CDC25A was analyzed by western blot. HSP60 was used as a loading control 
between 24 and $48 \mathrm{~h}$ in scratch-wounded NHK, was totally prevented in cells when miR-483-3p de novo synthesis was inhibited by LNA-483-3p (Figure 3d). In vivo wound-healing experiments performed on mouse tail showed that the expression of CDC25A decreased in epidermis when miR483-3p was strongly induced (Supplementary Figure S4).

These data establish CDC25A and CDK4 as bona fide target genes of miR-483-3p, which could have a critical role in the mediation of the anti-proliferative effect of miR-483-3p.

Impact of CDK4 and CDC25A invalidation on cell cycle progression. SiRNA invalidation of CDK4 (or CDK6) did not alter the serum-stimulated cell cycle progression (Figures $4 \mathrm{a}$ and $\mathrm{b}$ ). In contrast, the simultaneous invalidation of CDK4 and CDK6 blocked the cell cycle in early G1 (Supplementary Figure S5). These observations indicate that only downregulation of CDK4 by miR-483-3p is not sufficient by itself to inhibit $\mathrm{pRB}(\mathrm{S} 780)$ phosphorylation.

The invalidation of CDC25A led to different results. When CDC25A was downregulated using siRNA, HaCaT cells remained blocked in $\mathrm{G} 1$ after serum addition (61 versus $37 \%$ in G0/G1 phase) (Figure 4a). Interestingly, the silencing of CDC25A did not impair the stimulation of CCND1 but markedly blocked the induction of CCNE and CCNA, as well as the $\mathrm{pRB}(\mathrm{S} 780$ ) phosphorylation (Figure $4 \mathrm{~b}$ ), demonstrating that, like miR-483-3p, the suppression of CDC25A led to the inhibition of the CCND-associated kinases activity.

CDC25A ectopic expression rescues keratinocytes from miR-483-3p anti-proliferative effect. We selected HaCaT cell lines that stably express WT or catalytically inactive (C431S) forms of CDC25A. ${ }^{23}$ These two CDC25A variants lack the $3^{\prime}-U T R$, and are thus insensitive to miR-483-3p. Upon FCS, miR-483-3p did not inhibit the cell cycle progression of HaCaT transfected with WT-CDC25A, whereas it blocked control cells or catalytically inactive CDC25A-transfected cells in G1 (Figures 4c and d), strongly suggesting that CDC25A indeed participated to the antiproliferative effect of miR-483-3p.

The functional impact of CDC25A repression with regard to the anti-proliferative action of $\mathrm{miR}-483-3 p$ was finally demonstrated using a target protection approach. ${ }^{24}$ Two modified oligonucleotides ( $\mathrm{TSB}_{\mathrm{CDC} 25 \mathrm{~A}-1}$ and $\mathrm{TSB}_{\mathrm{CDC} 25 \mathrm{~A}-2}$ ) complementary to the miR-483-3p-binding sites of CDC25A, thus preventing the invalidation of CDC25A by miR-483-3p, were transfected in HaCaT cells and NHK (Figures $4 \mathrm{e}-\mathrm{g}$ ).

Upon FCS, CDC25A protection by $\mathrm{TSB}_{\mathrm{CDC} 25 \mathrm{~A}-1+2}$ led to a significant reduction in the number of cells blocked in $\mathrm{G} 1$ by miR-483-3p treatment (Figure $4 \mathrm{e}$ ). $\mathrm{TSB}_{\mathrm{CDC} 25 \mathrm{~A}-1+2}$ also abrogated the inhibitory effect of miR-483-3p on CCNE and CCNA expression, and pRB(S780) phosphorylation (Figure 4f).

In injured NHK, CDC25A protection significantly prevented post-mitotic cell cycle exit and maintained G1/S phase progression in the next cycles, as demonstrated by the sustained CCNE and CCNA expression, and pRB(S780) phosphorylation $72 \mathrm{~h}$ after injury (Figure $4 \mathrm{~g}$ ). $\mathrm{TSB}_{\mathrm{CDC} 25 \mathrm{~A}-1+2}$ also decreased the stimulation of early differentiation markers such as NICD, KRT1 and KRT10, and maintained TP63 expression, arguing for a delayed program of keratinocyte differentiation.

MiR-483-3p potentiates tyrosine phosphorylation on CDK6 and CDK4. The activity of the CCND-CDK6/4 complexes can be inhibited by agents that increase the phosphorylation of their tyrosine residues, notably when the CDK-specific tyrosine phosphatase CDC25A is inhibited. ${ }^{25,26}$ We analyzed the impact of miR-483-3p overexpression and CDC25A invalidation on the tyrosine phosphorylation status of CDK6 and CDK4 in HaCaT cells (Figure 5a, and Supplementary Figure S6). CDK6 immunoprecipitations coupled to anti-phosphotyrosine immunoblotting demonstrated that CDK6 was tyrosine-phosphorylated in FCSstarved HaCaT cells and strongly dephosphorylated upon FCS stimulation (Figure 5a). MiR-483-3p transfection markedly increased the basal tyrosine phosphorylation of CDK6 and strongly inhibited its dephosphorylation after FCS addition. Similarly, CDK6 remained tyrosine-phosphorylated upon serum stimulation in $\mathrm{HaCaT}$ cells invalidated for CDC25A, whereas it was dephosphorylated by $75 \%$ in cells treated with a control siRNA. SiRNA invalidation of CDC25A also potentiated the tyrosine phosphorylation of CDK4 and prevented its dephosphorylation when cells were stimulated with FCS (Supplementary Figure S6A).

CDC25A has a major role in the assembly of CCND-CDK4/6 complexes. The tyrosine phosphorylation of CDK6 and CDK4 inhibits their kinase activity, ${ }^{25,26}$ but the mechanisms underlying this inhibition have been incompletely elucidated.

Interestingly, we observed that the amount of CDK6 bound to CCND1 and CCND3 was markedly decreased in quiescent and FCS-stimulated HaCaT cells transfected with miR-483-3p or siCDC25A (Figure 5b). Incidentally, we noticed that CCND3 does not constitutively associate with CDK6 in quiescent cells, despite its high level of expression (Figure $5 b$ ).

It is worth noting that miR-483-3p overexpression did not preclude the formation of CCND1-CDK6 complexes in $\mathrm{HaCaT}$ cells that expressed an enzymatically active form of

\footnotetext{
Figure 4 CDC25A invalidation is essential for miR-483-3p cell cycle inhibition. (a and $\mathbf{b}$ ) Effect of CDK4, CDK6, CDC25A or control (Ctl) siRNAs on HaCaT cell cycle distribution by FACS (a), and western blot (b) analyses. Cells were synchronized in G0/G1 by FCS starvation and released into the cell cycle for 12 or $24 \mathrm{~h}$ by FCS addition. (c and d) Effect of miR-483-3p on the cell cycle of HaCaT cells stably expressing an empty pcDNA3 vector (Mock), WT-CDC25A or a phosphatase 'dead' C431S-CDC25A variant. Cells were synchronized, stimulated and analyzed as described in (a and $\mathbf{b}$ ). (d) MK2 was used as a positive control of miR-483-3p effect. (e and f) Effect of CDC25A target site blockers on miR-483-3p-induced cell cycle blockade. HaCaT cells were cotransfected with $50 \mathrm{nM}$ of CDC25A TSB (TSB $\left.\mathrm{CDC}_{\mathrm{C} 25 \mathrm{~A}-1} ; \mathrm{TSB}_{\mathrm{CDC} 25 \mathrm{~A}-2}\right)$ or TSB $\mathrm{CHt}_{\mathrm{C}}$ in the presence of $5 \mathrm{nM}$ of pre-miR-NC or pre-miR-483-3p and then synchronized, stimulated and analyzed as described in (a-b). (g) Effect of CDC25A TSB on scratch-woundinduced miR-483-3p. NHK were transfected with $10 \mathrm{nM}$ of $\mathrm{TSB}_{\mathrm{CDC} 25 \mathrm{~A}-1+2}$ or $\mathrm{TSB}_{\mathrm{Ct}}$, grown to confluence, scratch wounded and harvested at the indicated time points to analyze cell cycle regulators and differentiation markers by western blot. (a, c, e) Data point out the percentage of HaCaT cells in G0/G1, S and G2/M phases after FCS-deprivation or $24 \mathrm{~h}$ after FCS-addition, and are representative of four independent experiments
} 
CDC25A (Figure 5c), demonstrating that the enzymatic activity of CDC25A was required for proper association between CDK6 and CCND1.

a

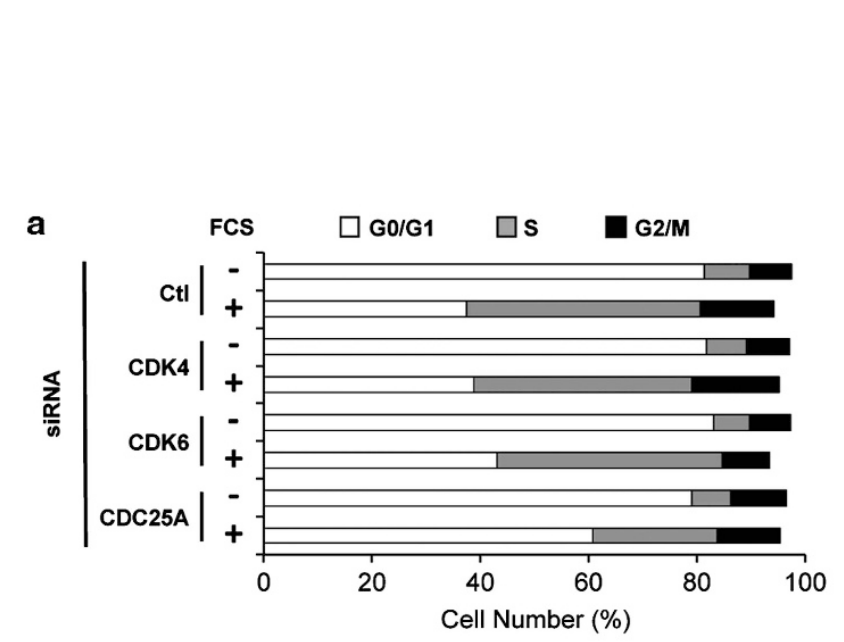

Loss of CDC25A also increased the tyrosine phosphorylation level of CDK4 and blocked its capacity to assemble with CCNDs (Supplementary Figures S6A and B).

b SiRNA $\frac{\text { Ctl }}{01124} \frac{\text { CDK4 }}{01224} \frac{\text { CDK6 }}{01224} \frac{\text { CDC25A }}{01224}$
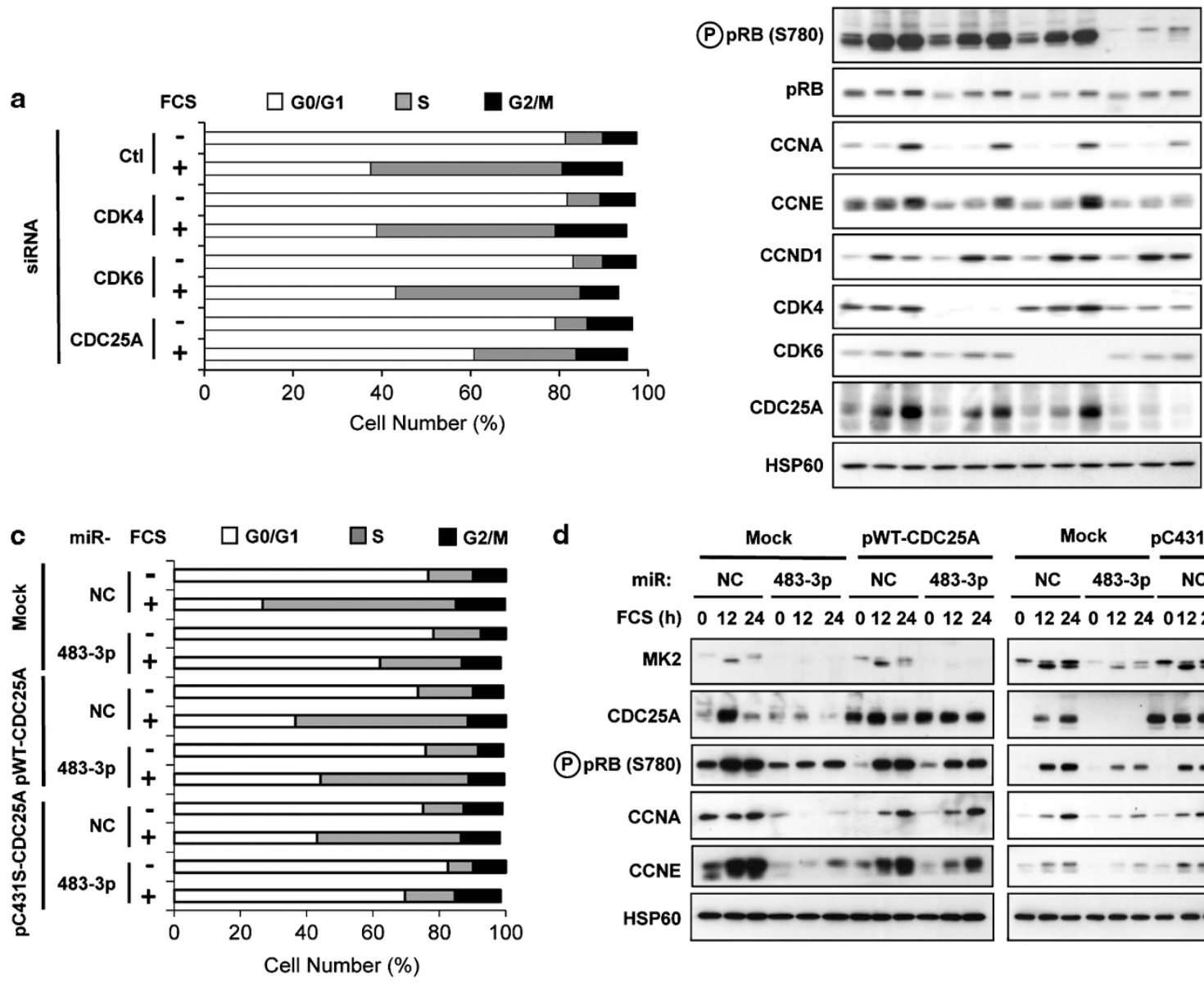

d<smiles>CCCCC[SiH2]</smiles>

miR: $\frac{\text { Mock }}{\text { NC } 483-3 p} \frac{\text { pWT-CDC25A }}{\text { NC } 483-3 p} \frac{\text { Mock }}{\text { NC } 483-3 p} \frac{\text { pC431S-CDC25A }}{\text { NC } 483-3 p}$

FCS (h) $\overline{01224} \overline{01224} \overline{01224} \overline{01224} \overline{01224} \overline{01224} \overline{01224} \overline{01224}$

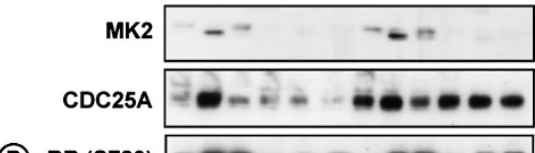

PPRB (S780)
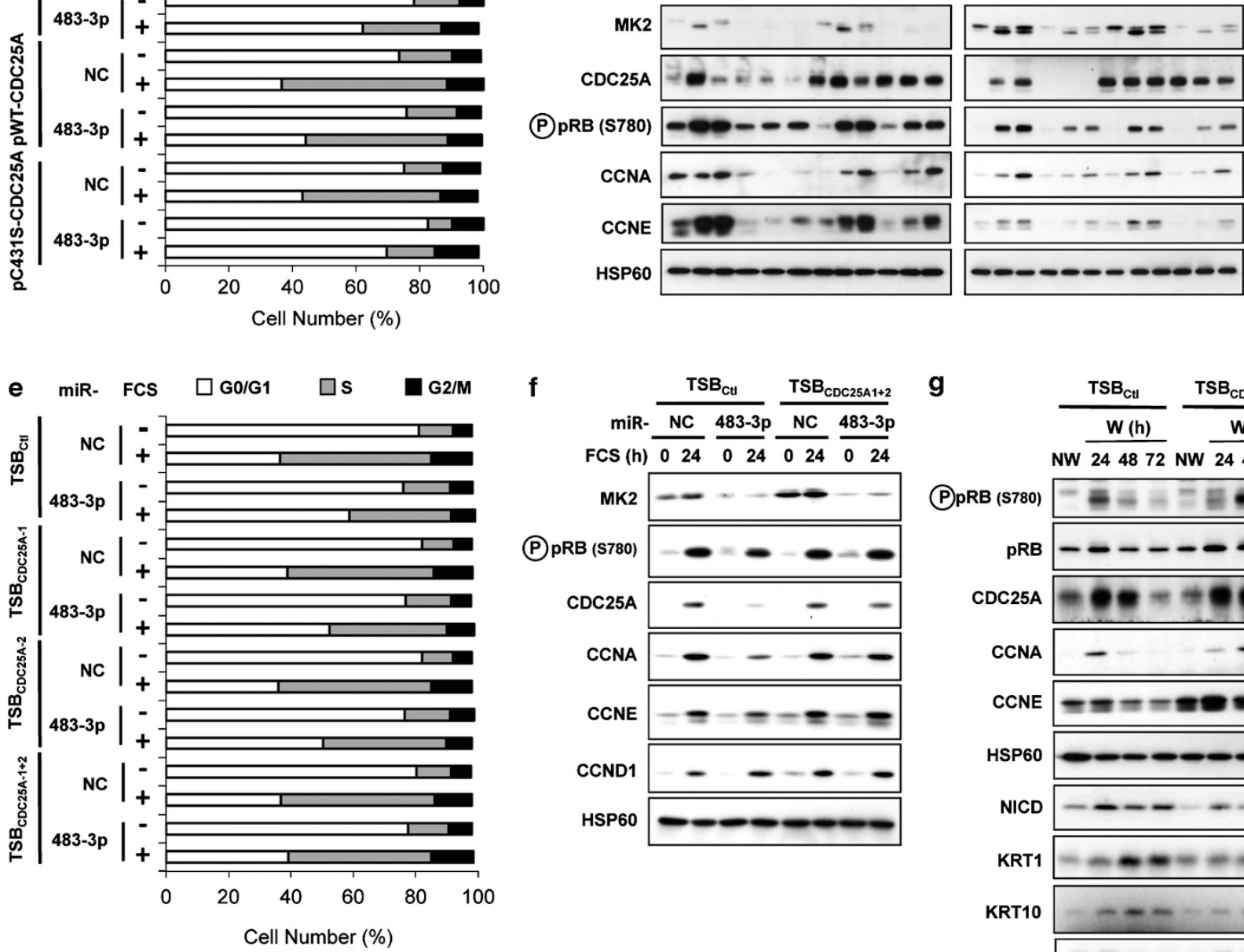

$\mathbf{f}$

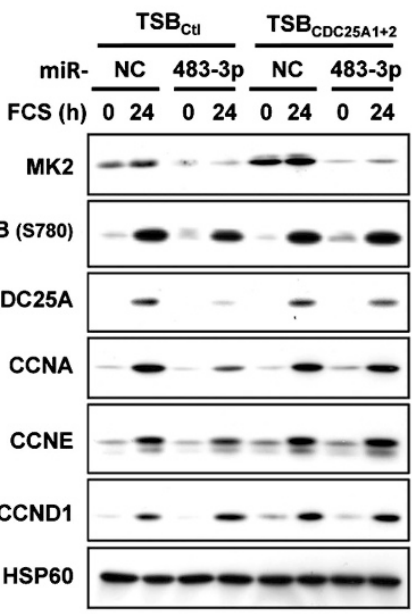

g

$\frac{\text { TSB }_{\mathrm{Ctl}}}{\mathrm{NW} \frac{\mathrm{W}(\mathrm{h})}{244872}} \frac{\text { NSB }_{\mathrm{CDC25A1+2}}}{\frac{W(h)}{244872}}$

(P)pRB (5780)
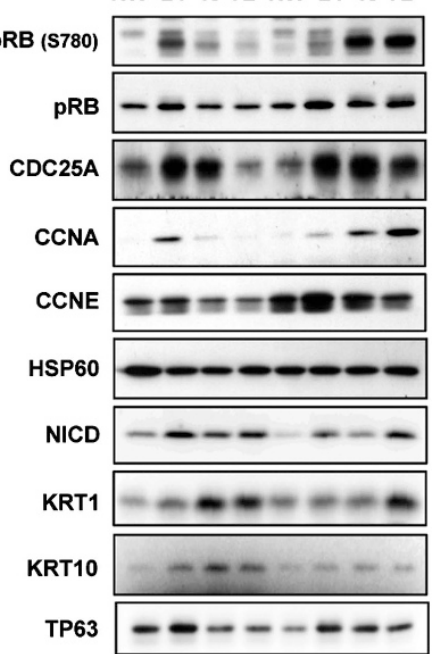
Thus, miR-483-3p-dependent inhibition of CDK6 and CDK4 activities results, at least in part, from a defective association with CCNDs. CDK6 and CDK4 dephosphorylation by CDC25A controls these associations.

Interestingly, we noticed in cells treated with miR-483-3p, siCDC25A or a pharmacological inhibitor of CDC25A, that upon FCS stimulation, the neosynthesized cyclin D1 translocated into the nucleus, whereas CDK4/CDK6 were retained in the cytosol (Supplementary Figures S6C, S7A-B and S8). In addition, this miR-483-3p-mediated inhibition of CDK6 nuclear import was efficiently reverted by the expression of WT-CDC25A or CDC25A-target-site blockers (Supplementary Figures S7C and D).

The CDK6(Y24) phosphorylation prevents the formation of CCND-CDK6 complexes. We then investigated the role of CDK6(Y24) phosphorylation in formation of CCND-CDK6 complexes because CDK6 is not a direct target of miR-483-3p and, in contrast to CDK4, its expression is not affected by the presence of miR-483-3p.

Y24 is the only CDK6 tyrosine residue reported so far for being phosphorylated, thus inhibiting the kinase activity of CCND-CDK6 complexes. ${ }^{25,26}$ A non-phosphorylatable Y24F mutant of CDK6, ectopically expressed in HEK293 cells, associated much better with CCNDs than the WT-CDK6 form (Figure 6a). Moreover, CCND-CDK6 association was not hampered by miR-483-3p in Y24F-CDK6-transfected HEK293 cells, although it was totally inhibited in cells transfected with WT-CDK6 (Figure 6a). Similarly, in quiescent and FCS-stimulated HaCaT cells transfected with Y24FCDK6 or WT-CDK6, the amount of CCNDs associated with Y24F-CDK6 was higher than that with WT-CDK6 (Figure 6b). Overexpression of miR-483-3p strongly impaired the association of CCNDs with WT-CDK6, whereas it did not affect the formation of CCND-Y24F-CDK6 complexes, indicating that: (1) phosphorylation of CDK6 Y24 residue indeed antagonizes the binding with CCND, and (2) the inhibitory effect of miR483-3p on CCND-CDK6 assembly routed through the dephosphorylation of the CDK6 Y24 residue.

BrdU incorporation performed in exponentially growing HEK293 cells (Figure $6 \mathrm{c}$ ) and in FCS-stimulated HaCaT keratinocytes (Figure 6d) indicated that cells transfected with Y24F-CDK6 were able to enter in $S$ phase in the presence of miR-483-3p, whereas cells expressing the WT form were strongly inhibited.

\section{Discussion}

This manuscript reports a novel cell cycle regulatory mechanism involved in the proliferation arrest that follows contact inhibition. This mechanism is controlled by miR-483-3p, which is specifically upregulated when keratinocytes sheets, disrupted by injury, recover confluence. We demonstrate here the downregulation of CDC25A phosphatase by miR-483-3p, and how this invalidation interferes with the cell cycle progression.

Our results show that the ectopic expression of miR-483-3p blocks the cell cycle progression of keratinocytes stimulated by serum or injury in early G1 phase. Elsewhere, all approaches that antagonize the neo-synthesis of miR-483-3p following injury compromised the exit of cell cycle driven by confluence and facilitated the G1/S phase progression into the next cycles. These observations emphasize the important role of miR-483-3p in the control of quiescence. We also observed that preventing miR-483-3p action delayed the appearance of early differentiation markers, indicating that miR-483-3p is mandatory for the cell-growth arrest that precedes commitment of keratinocytes to terminal differentiation.

Our molecular analyses established that miR-483-3p interrupts the cell cycle progression in $\mathrm{G} 1$ by inhibiting the activity of the CCND-dependent kinases CDK4 and CDK6. This inhibition mostly results from an enhanced tyrosine phosphorylation of CDK4 and CDK6, due to the direct invalidation of CDC25A by miR-483-3p. The $\mathrm{Y} 17$ and Y24 residues of CDK4 and CDK6 are equivalent to the Y15's of CDK1/CDK2 that have a key role in the regulation of these kinases. When phosphorylated, Y17 and Y24 inhibit the kinase activity of CDK4 and CDK6. ${ }^{25,26}$ Both residues are located within the conserved 'glycine-rich' motif (GXGXYG) of CDK6/4 required for ATP binding. Their phosphorylation is controlled by a balance between kinases, such as Src family members, and CDC25 phosphatases. ${ }^{27,28}$ From the three CDC25 isoforms (CDC25A, CDC25B and CDC25C) present in human cells, which activate CDKs at different phases of the cell cycle through dephosphorylation of their 'glycin-rich motifs', ${ }^{28,29}$ CDC25A is the only member required for the control of G1/S CDK's activities. Its dominant role in cell cycle control appears consistent with a high incidence of upregulation in cancer. ${ }^{29,30}$ So far, the impact of CDC25A in the control of G1 progression was mostly attributed to its effect on CCNE-CDK2 or CCNA-CDK2 complexes. ${ }^{31}$ Here, we demonstrate that the cell cycle blockade consecutive to CDC25A inhibition by miR-483-3p, siRNAs or pharmacological drugs, precedes CCNE induction and results from the inhibition of the kinase activity of CCND-CDK4/6 complexes. Similar observations have also been reported in TGF $\beta$ - or DNA damage-induced G1 arrest, ${ }^{25,26}$ as well as in cells lacking plk3. ${ }^{32}$ However, the molecular connection between the absence of CDC25A and the cell cycle blockade in very early $\mathrm{G} 1$ has never been elucidated so far.

Structural studies and enzymatic analyses demonstrated that $\mathrm{Y} 15$ phosphorylation participates in the enzymatic

Figure 5 miR-483-3p prevents CDK6 tyrosine dephosphorylation and assembly with CCND. (a) Effect of miR-483-3p and CDC25A siRNA on the tyrosine phosphorylation status of CDK6. HaCaT cells transfected with pre-miR-483-3p or si-CDC25A were synchronized by serum starvation and then stimulated with FCS for $12 \mathrm{~h}$. miR-NC and siCtl refer to control miRNA and siRNA. Tyrosine phosphorylation (P-Tyr) was detected by western blot after immunoprecipitation with anti-CDK6 antibodies. Histograms represent the quantification of the P-Tyr signal normalized for CDK6. Lower panels represent the efficiency of miR-483-3p- and siRNA-mediated CDC25A invalidation. (b) Effect of miR483-3p and CDC25A siRNA on the formation of CCND-CDK6 complexes. Lysates from HaCaT cells treated as described in (a) were immunoprecipitated with anti-CCND1, anti-CCND3 or anti-CDK6 antibodies and then blotted with Phospho-pRB(S780), CCND1, CCND3 and CDK6 antibodies. (c) HaCaT cells constitutively expressing an empty vector (Mock), WT-CDC25A or the C431S-CDC25A variant were transfected with pre-miR-NC or pre-miR-483-3p and treated as described in (a). CCND1 was immunoprecipitated, and CCND1-bound CDK6 measured by immunoblotting. Lower panel shows the analysis of CDC25A levels in total cell lysates 
a
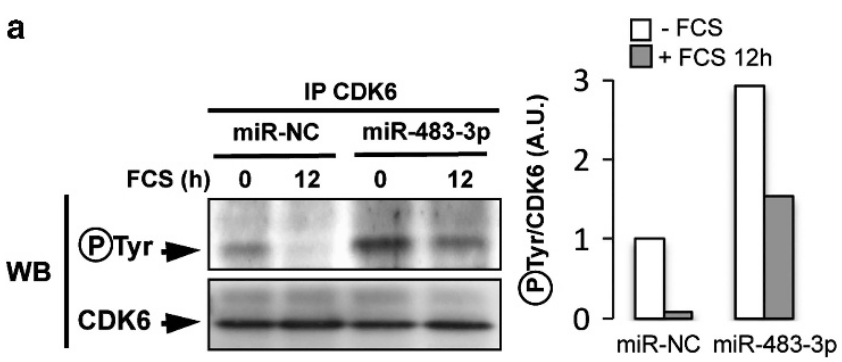

cell lysates

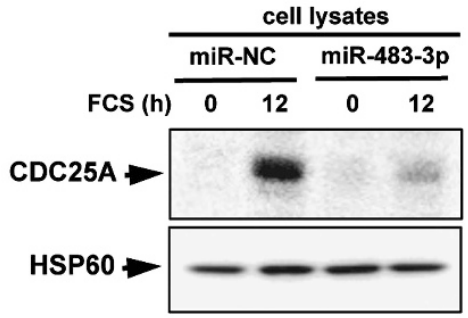

b

IP CCND1

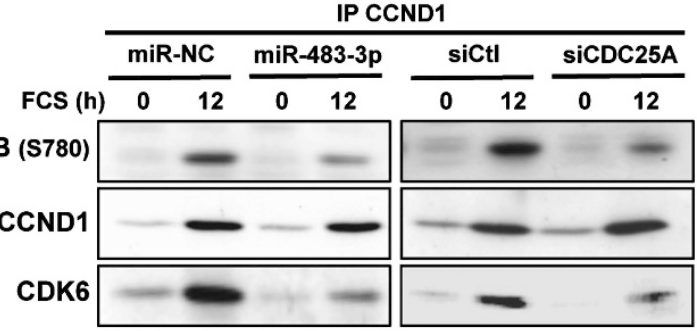

IP CCND3

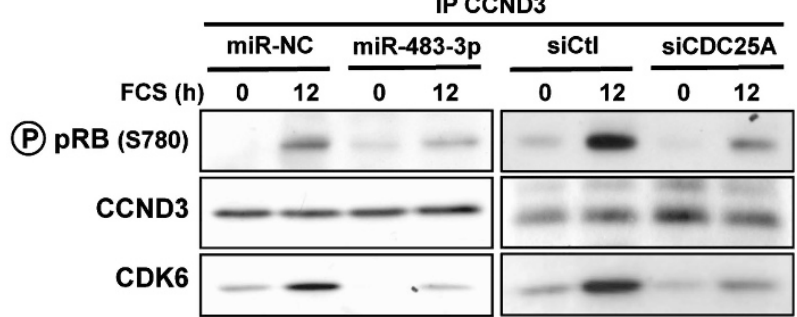

IP CDK6

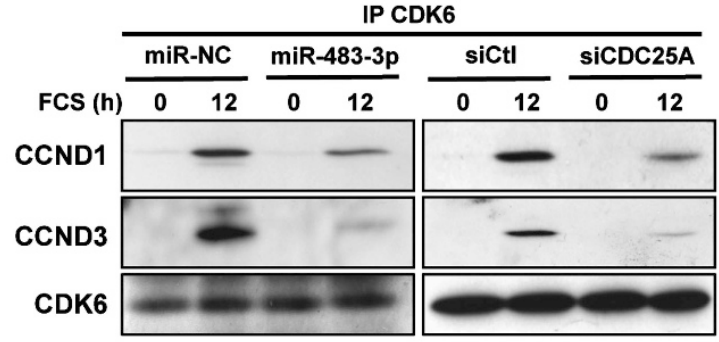

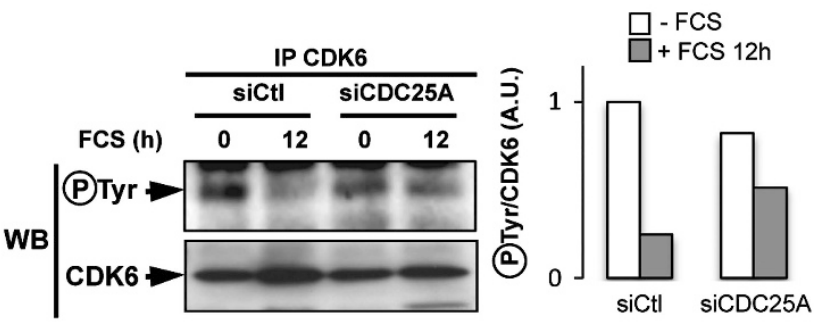

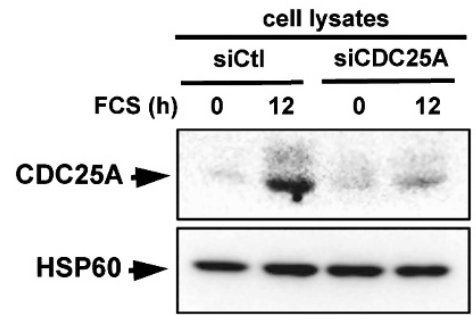

C

C IP CCND1
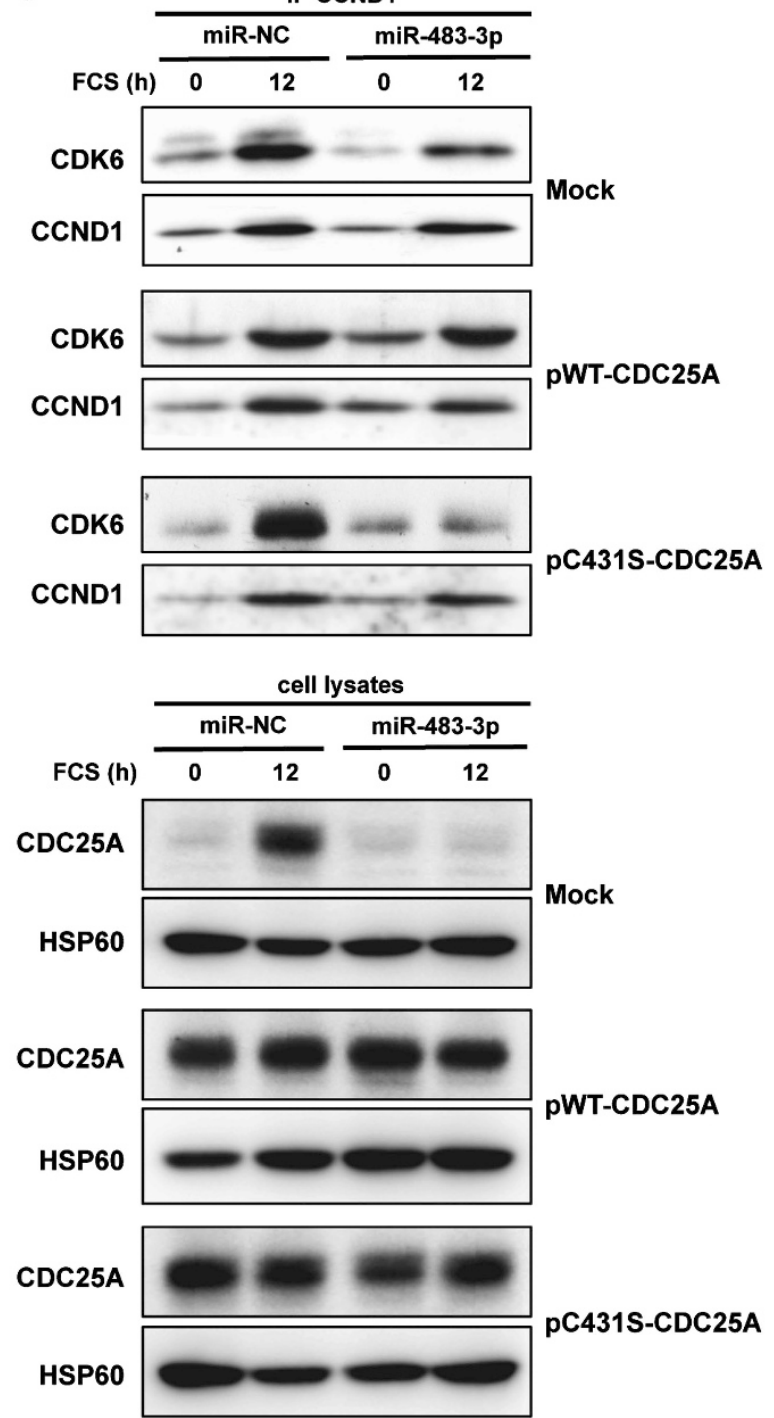
a
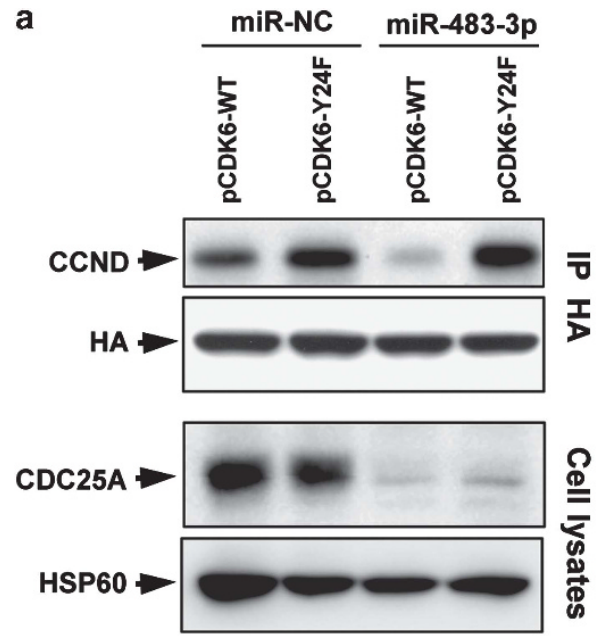

c

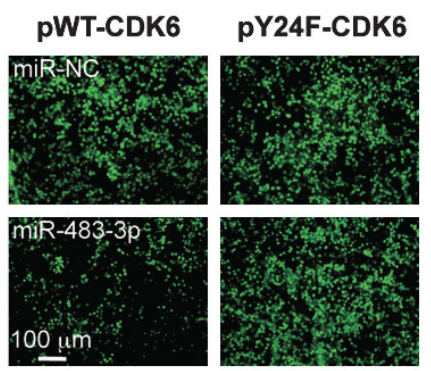

asynchronous

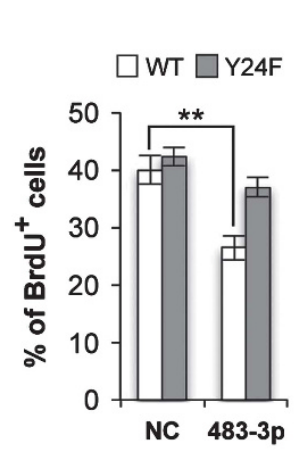

b
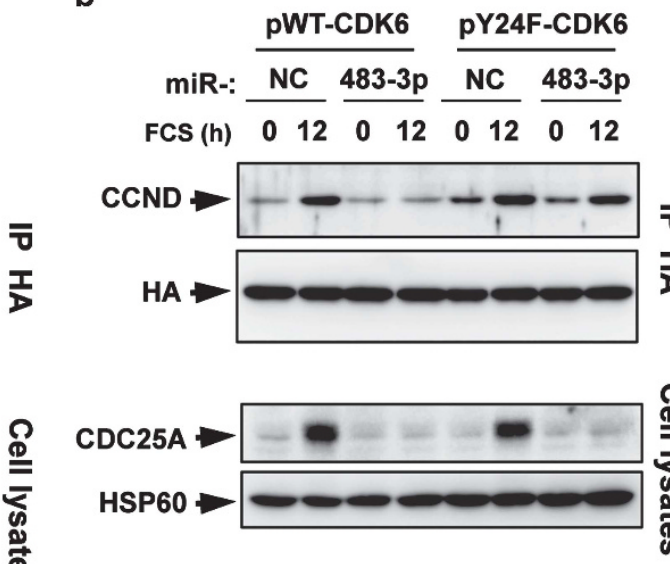
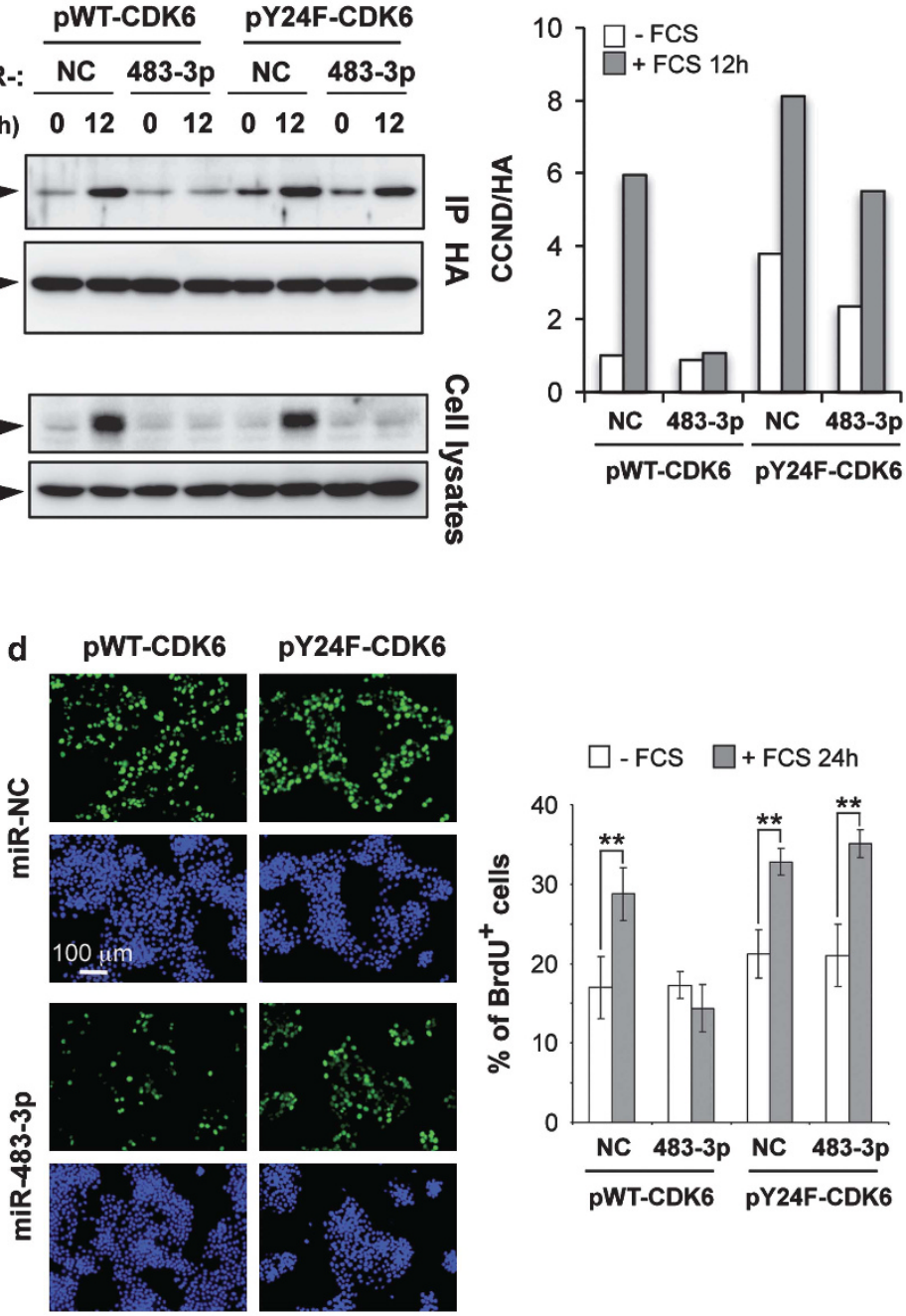

+ FCS 24h

Figure 6 CDK6(Y24) phosphorylation inhibits CCND-CDK6 assembly and prevents cell cycle progression. (a and b) HEK293 and HaCaT cells were transfected with expression vectors for HA-tagged WT-CDK6 or Y24F-CDK6 in the presence of pre-miR-NC or pre-miR-483-3p. Lower panels show the efficiency of miR-483-3p-mediated CDC25A invalidation. (a) In HEK293, CDK6 was immunoprecipitated $48 \mathrm{~h}$ after transfection using an anti-HA antibody, and the associated CCNDs were measured by immunoblot using a pan-CCND antibody. (b) Transfected HaCaT cells were synchronized in G0/G1 by FCS starvation and then released for $12 \mathrm{~h}$ by FCS addition. CCND expression in anti-HA immunoprecipitates was analyzed as in (a). Histograms show the quantification of CCND normalized to the level of HA-CDK6. (c and d) Effect of CDK6 Y24F mutation on cell cycle progression. BrdU incorporation was measured in exponentially growing HEK293 (c), and in synchronized HaCaT (d) cells transfected like in $(\mathbf{a}$ and $\mathbf{b})$. Nuclei were stained with DAPI. Data are means \pm S.D. from eight independent microscope fields. Statistically significant differences are indicated $\left({ }^{* *} P<0.001\right)$

inhibition of CDK1 and CDK2 by reducing ATP binding and/or affinity for their substrate. ${ }^{33-35}$ However, to date, the mechanisms underlying the inhibition of CCND-dependent kinases by tyrosine phosphorylation have not been elucidated. Here we bring direct new evidences that phosphorylation of Y24 inhibits CDK6 activities by preventing its association with CCNDs.

The mechanisms involved in the regulation of CCND-CDK4/6 association have been the matter of intense speculations. ${ }^{36}$ In quiescent fibroblasts, ectopically expressed CDK4 and CCND3 do not spontaneously associate. Their assembly depends on a serum-stimulated event. ${ }^{37}$ Here we demonstrate that the Y24 phosphorylation of CDK6 is likely the inhibitory signal preventing this association, as the Y24F-CDK6 better associates with CCNDs than WT-CDK6, even in resting cells. While additional post-traductional modifications such as the
CAK-mediated CDK6 T178 phosphorylation represents other mechanisms for the holoenzyme activation, ${ }^{38}$ our data emphasize the importance of the assembly step of complexes in the activity of the holoenzyme.

The GXGXYG domain of CDK4 containing the Y17 residue is a highly flexible region that is also involved in the binding of CDC37/HSP90. These chaperones have been shown to regulate CDK4-CCND1 association. Indeed, mutations of the G15 or G18 residues precluded CDC37-CDK4 complex formation and greatly reduced the binding of CDK4 to CCND1, ${ }^{39}$ indicating that slight structural modifications within the 'glycine-rich' motif are detrimental for the assembly of CCND-CDK4 complexes. It is highly conceivable that the negative charges brought by the phosphorylation of Y24 in the CDK6 GXGXYG domain impose conformational changes or generate steric hindrance that could interfere with the binding 


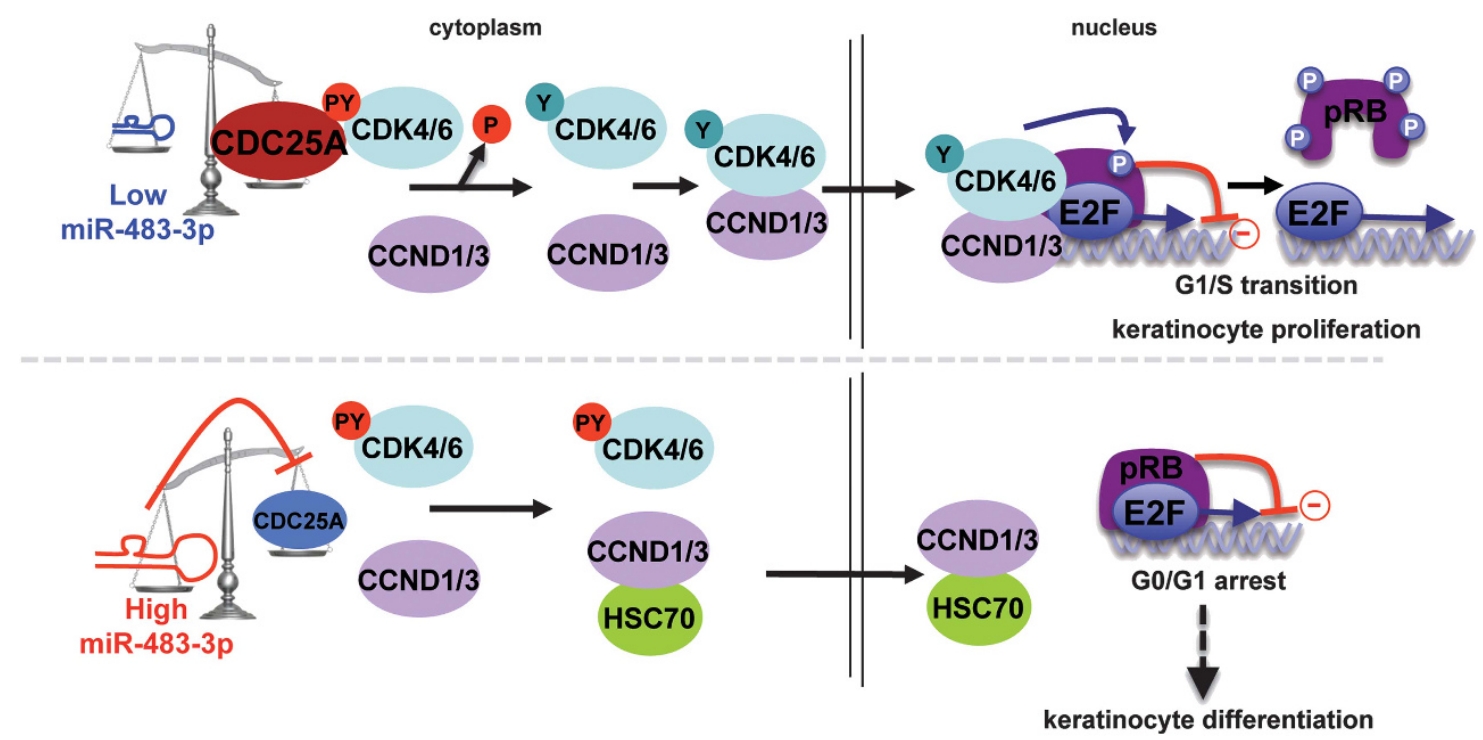

Figure 7 Hypothetical model summarizing the molecular bases of miR-483-3p anti-proliferative effect. At the end of wound healing, the miR-483-3p-mediated invalidation of CDC25A prevents the dephosphorylation of the CDK4/6 Y17/24 residues, which impairs the formation of CCND1/3-CDK4/6 complexes, hampering their activation and blocking cells in early $\mathrm{G} 1$, which could favor keratinocyte commitment to terminal differentiation

of CCNDs. Future X-ray crystallographic studies should clarify this question.

CDC25A is exquisitely regulated during the cell cycle. It is upregulated from $\mathrm{G} 1$ to $\mathrm{G} 2$ phase then downregulated when cells enter mitosis, and stimulated again in the next $\mathrm{G} 1$ phase. The mechanisms whereby CDC25A is repressed are complex. $^{30}$ For a long time, the degradation of CDC25A by an ubiquitin-mediated proteolysis was considered as the unique mechanism of CDC25A turnover. ${ }^{28}$ However, the recent identification of several miRNAs targeting CDC25A (miR15a, miR-16, miR-21, miR-125b, miR-424, miR-449a/b, miR503 , let-7 $)^{5-12}$ clearly establishes that the miRNA-dependent repression of CDC25A also corresponds to a significant component of cell cycle control.

In this context, our results can explain how CDC25A invalidation has an impact on cell cycle, by showing that CD25A silencing precludes the association between CDK4/6 and their partner cyclins. They provide an adequate framework to explain the anti-proliferative effects reported for all others CDC25A-targeting miRNAs.

Our data are summarized in Figure 7. The upregulation of miR-483-3p, either by ectopic expression or after keratinocyte injury, directly targets CDK4 and CDC25A. Invalidation of CDK4 has no impact by itself on the cell proliferation, but invalidation of CDC25A prevents the dephosphorylation of CDK6 (Y24) and CDK4 (Y17) residues, and impedes their association with CCNDs. This inhibition impairs the activation of CCND-dependent kinases, prevents the phosphorylation of pRB and blocks cells in early $\mathrm{G} 1$ upstream the transcriptional induction of CCNE.

The anti-proliferative effect of miR-483-3p in wound healing is in accordance with its decreased expression in the hyperproliferative epidermis of patients suffering from atopic dermatitis and psoriasis, ${ }^{40,41}$ and in gastric, nasopharyngeal and hepatocellular carcinomas. ${ }^{42-45}$ However, there is currently no functional evidence of any physiopathological implication of miR-483-3p downregulation in these processes. Conversely, miR-483-3p is overexpressed in cancers associated with biallelic expression of its host gene IGF2 due to LOI of the IGF2/H19 locus. ${ }^{16,17,46-48}$ In these tumors, miR483-3p was proposed to have an anti-apoptotic oncogenic role by targeting the BBC3/PUMA gene, thus favoring cell survival. ${ }^{16,17}$ In keratinocytes, we previously observed that PUMA expression was not modulated by miR-483-3p, ${ }^{13}$ indicating that such a gene targeting could be cell-type specific or could depend on a defined biological process. In the same way, one can hypothesize that the apparent discrepancy between the overexpression of $\mathrm{miR}-483-3 p$ in these cancers and our data on its anti-proliferative properties could result from the dysregulation of several oncogenic or tumor-suppressive pathways controlling the cell cycle in these tumors.

In conclusion, this study shed some light on an important mechanism of regulation of CCND-dependent kinases' activity. We demonstrate that the induction of CCNDs is not sufficient per se to launch cells into the cell cycle, but also requires that CDK4/6 are prone to interact with them. The formation of the holoenzyme, which precedes the activation of the kinases, requires at least an additional step, that is, the dephosphorylation by CDC25A at key tyrosine residues located in the 'glycine-rich' domain of CDK4/6. Besides its well documented effect on CCNE-CDK2 at the G1/S boundary, CDC25A thus appears also as a key regulator of the very early steps of the cell cycle by targeting CDK4/6, heavily controlled by many miRNAs, including miR-483-3p.

\section{Materials and Methods}

Cell culture and wounding protocol. NHK isolated from healthy neonatal foreskins were cultured on a feeder layer of lethally irradiated 3T3-J2 fibroblasts and wounded using a homemade 'helicoidal scarificator' as previously described. ${ }^{13}$ In transfection experiments, NHK were seeded and transfected in defined KGM2 medium (Lonza, Basel, Switzerland). Feeder cells and fresh medium were added $24 \mathrm{~h}$ after transfection. The COS-7 monkey kidney cell line 
(ATCC; Rockville, MD, USA) and the human HaCaT-immortalized keratinocytes (gift from Dr. P Boukamp, Heidelberg, Germany) were grown in DMEM containing $10 \%$ FCS (Perbio Science AB, Helsingborg, Sweden). In some experiments, $\mathrm{HaCaT}$ cells were cultured in the presence of CDC25A inhibitor II (Calbiochem, Merck, Darmstadt, Germany).

Keratinocyte transfection. Pre-miRNAs (pre-miR-483-3p and negative control pre-miR-NC1), anti-miRNAs oligonucleotides (LNA-159s and LNA-483-3p), custom designed target site blockers LNA oligonucleotides (TSB ${ }_{C D C 25 A-1:}$

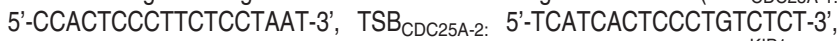
$\mathrm{TSB}_{\mathrm{Ct}}$ ) and ON-Targetplus siRNAs (for CDK4, CDK6, CDC25A, p27 ${ }^{\mathrm{KIP} 1}$ and irrelevant control (Ctl)) were purchased from Ambion (Austin, TX, USA), Exiqon (Vedbaek, Denmark) and Dharmacon (Thermo Fisher Scientific, Courtaboeuf, France), respectively. NHK and HaCaT cells were plated and transfected $24 \mathrm{~h}$ later at 30-50\% confluency, with pre-miRNAs (10 nM), anti-miRNAs (20 nM), siRNAs $(25 \mathrm{nM})$ or TSB $(10-50 \mathrm{nM})$ using Lipofectamin RNAiMAX reagent (Life Technologies, Carlsbad, CA, USA). Transfection efficiency, evaluated by FACS analysis using a FAM-labeled pre-miR-NC, ranged between 60 and 90\% depending on cell type and cell density. HaCaT cells stably expressing WT-CDC25A or C431S-CDC25A 'phosphatase dead' variant were generated by transfection of pcDNA3.1 construct (gift from Dr. S Manenti, Toulouse, France) $)^{23}$ using Fugene reagent (Promega, Madison, WI) and selected in medium containing $1 \mathrm{mg} / \mathrm{ml}$ of G418 (Life Technologies). Reverse transfection of HaCaT cells with pWT-CDK6 and pY24F-CDK6 was done using the Fugene reagent.

Cell cycle analysis. Exponentially growing $\mathrm{HaCaT}$ cells were transfected at $30 \%$ confluency. The next day cells were synchronized by serum starvation for $35 \mathrm{~h}$ and then released to enter cell cycle by the addition of $10 \%$ FCS. Cells were collected at different time and analyzed by western blotting or flow cytometry. Briefly, cells were fixed with $70 \%$ ethanol and stained with propidium iodide solution (Sigma St. Louis, MO, USA) containing RNAse A. Data were acquired on a FACScalibur flow cytometer and analyzed using CellQuest Pro software (Becton-Dickinson, Franklin Lakes, NJ, USA).

Antibodies. Rabbit polyclonal antibodies (pAb) to CCND, CCNA, p21 ${ }^{\mathrm{CIP} 1}$, p2 $7^{\mathrm{KIP} 1}$, CDK4, CDK6 and anti-HSP60 goat pAb, and mouse monoclonal antibodies (mAb) to CCND1, CCND3, CCNE and CDC25A were purchased from Santa Cruz Biotechnology (Santa Cruz, CA, USA). Rabbit pAb to p15, MK2 and Phospho-pRB (S780) were from Cell signaling Technology (Danvers, MA, USA). Anti-phosphoTyrosine (4G10) and anti-pRB mouse mAbs were from Millipore (Billerica, MA, USA). Anti-HA mouse mAb was from Sigma Aldrich. The secondary peroxidase-conjugated pAb were from Dakopats (Hamburg, Germany).

Immunofluorescence. HaCaT cells were grown on glass coverslip, fixed with methanol for $10 \mathrm{~min}$ and labeled with anti-CDK6 or anti-CDK4 pAbs and anti-CCND1 mAb. Secondary antibodies coupled with Alexa-488 or Alexa-594 (Life Technologies) were used at 1:500. Cells were stained with DAPI (Sigma) and coverslips mounted on glass slides with mounting medium.

Immunoprecipitation and immunoblot detection. Preparation of cell lysates, conditions for immunoprecipitation and immunoblotting have been described previously. ${ }^{49}$ For immunoprecipitations, $250-500 \mu \mathrm{g}$ of proteins were incubated with antibodies against CCND1, CCND3, CDK4 or CDK6 for $2 \mathrm{~h}$ at $4^{\circ} \mathrm{C}$. Immunocomplexes bound to PureProteome Protein G Magnetic Beads (Millipore) were washed four times with PBS $0.1 \%$ Tween 20 and twice with lysis buffer before resolving by SDS-PAGE.

miRNA and gene expression analyses by real-time qPCR. Total RNA was isolated with TRIzol reagent (Life Technologies) according to the manufacturer's instructions. Expression of mature hsa-miR-483-3p was evaluated using TaqMan MicroRNA Assays (Applied Biosystems, Foster City, CA, USA) and normalized to RNU44. For gene expression, RNAs were retro-transcribed with the Superscript II RT kit (Life Technologies) and PCR performed using SYBR Green Master mix II (Applied Biosystems). Gene expression levels were normalized to RPLP0 level. All reactions were done in triplicate using ABI PRISM 7900HT Sequence detection system (Applied Biosystems) and expression levels calculated using the comparative CT method $\left(2^{-\Delta \Delta C T}\right)$.
Plasmid constructs, site-directed mutagenesis and luciferase assays. NHK CDNA fragments corresponding to human CDK4, CDK6, and CDC25A 3'-UTR mRNAs were cloned into the Xhol and Notl restriction sites downstream from the Renilla luciferase gene of psiCHECK-2 vector (Promega) using the following primers: CDC25A-F 5'-GGAGGAGCATGTCCACTGAGT ATC-3', CDC25A-R 5'-GCCCCAGCTCCTTGGATGAGG-3', CDK4-F 5'-GTGC AGTCGGTGGTACCTGAG-3', CDK4-R 5'-GTGTCCAGAAGGGAAATGGCAGC-3', CDK6-F 5'-GCTGCTGACCAATTGTGCTGCC-3' and CDK6-R 5'-CCAACAAGG CAGTGTGTGGCAG-3'. Luciferase assays were performed in COS-7 cells as previously described. ${ }^{13}$ Mutagenesis of the putative miR-483-3p binding sites was performed using the QuickChange multisite-directed mutagenesis kit from Stratagene (Agilent, La Jolla, CA, USA) according to the manufacturer's protocol. Mutagenesis of $3^{\prime}$-UTRs was performed using the following primers, in which 'seed' interacting sequences are in boldface, and mutated bases are underscored:CDC25A-MUT1 5'-CCTGGGCATTAGGAGAAGGGTGAGCGTGCTGGC ATGGAC-3', CDC25A-MUT2 5'-TTTTAGAGACAGGGAGĀGĀAGTATCATCAC CGGTTG-3' and CDK4-MUT 5'-CCGGAGTGAGCAATGGT'GÁGCCTGCCA TGG-3'. CDK6-coding sequence (NM_001259.6) was amplified by PCR and cloned into $E$ CoRV/Xhol sites of pCDNA3.1 containing an additional HA tag at its $\mathrm{C}$-terminus. A single nucleotide mutation changing amino acid 24 from tyrosine to phenylalanine was introduced using QuickChange singlesite-directed mutagenesis kit from Stratagene.

Statistical analysis. Statistical evaluations were performed by Student's $t$-test for paired data, and data were considered significant at a $P$-value inferior to 0.05 .

\section{Conflict of Interest}

The authors declare no conflict of interest.

Acknowledgements. This work was supported by funds from the Association pour la Recherche sur le Cancer (ARC, Grant 5420). Thomas Bertero was a recipient of a doctoral contract from the Ministry of Higher Education and Research. Cécile Gastaldi was a recipient of a fellowship from INSERM/PACA region.

1. Schafer M, Werner S. Cancer as an overhealing wound: an old hypothesis revisited. Nat Rev Mol Cell Biol 2008; 9: 628-638.

2. Sherr CJ, Roberts JM. Living with or without cyclins and cyclin-dependent kinases. Genes Dev 2004; 18: 2699-2711.

3. Bueno MJ, Malumbres M. MicroRNAs and the cell cycle. Biochim Biophys Acta 2011; 1812: $592-601$.

4. Rissland OS, Hong SJ, Bartel DP. MicroRNA destabilization enables dynamic regulation of the miR-16 family in response to cell-cycle changes. Mol Cell 2011; 43: 993-1004.

5. Johnson CD, Esquela-Kerscher A, Stefani G, Byrom M, Kelnar K, Ovcharenko D et al. The let-7 microRNA represses cell proliferation pathways in human cells. Cancer Res 2007; 67: 7713-7722.

6. Lee SO, Masyuk T, Splinter P, Banales JM, Masyuk A, Stroope A et al. MicroRNA15a modulates expression of the cell-cycle regulator Cdc25A and affects hepatic cystogenesis in a rat model of polycystic kidney disease. J Clin Invest 2008; 118: 3714-3724.

7. Yang $X$, Feng $M$, Jiang $X$, Wu Z, Li Z, Aau $M$ et al. miR-449a and miR-449b are direct transcriptional targets of $\mathrm{E} 2 \mathrm{~F} 1$ and negatively regulate $\mathrm{pRb}-\mathrm{E} 2 \mathrm{~F} 1$ activity through a feedback loop by targeting CDK6 and CDC25A. Genes Dev 2009; 23: 2388-2393.

8. Wang P, Zou F, Zhang X, Li H, Dulak A, Tomko RJ Jr. et al. microRNA-21 negatively regulates Cdc25A and cell cycle progression in colon cancer cells. Cancer Res 2009; 69 : $8157-8165$.

9. Pothof J, Verkaik NS, van IW, Wiemer EA, Ta VT, van der Horst GT et al. MicroRNAmediated gene silencing modulates the UV-induced DNA-damage response. EMBO J 2009; 28: 2090-2099.

10. Shi L, Zhang J, Pan T, Zhou J, Gong W, Liu N et al. MiR-125b is critical for the suppression of human U251 glioma stem cell proliferation. Brain Res 2010; 1312: 120-126.

11. Sarkar S, Dey BK, Dutta A. MiR-322/424 and -503 are induced during muscle differentiation and promote cell cycle quiescence and differentiation by down-regulation of Cdc25A. Mol Biol Cell 2010; 21: 2138-2149.

12. Marcet B, Chevalier B, Luxardi G, Coraux C, Zaragosi LE, Cibois M et al. Control of vertebrate multiciliogenesis by miR-449 through direct repression of the Delta/Notch pathway. Nat Cell Biol 2011; 13: 693-699.

13. Bertero T, Gastaldi C, Bourget-Ponzio I, Imbert V, Loubat A, Selva E et al. miR-483-3p controls proliferation in wounded epithelial cells. FASEB J 2011; 25: 3092-3105. 
14. Ferland-McCollough D, Fernandez-Twinn DS, Cannell IG, David H, Warner M, Vaag AA et al. Programming of adipose tissue miR-483-3p and GDF-3 expression by maternal diet in type 2 diabetes. Cell Death Differ 2012; 19: 1003-1012.

15. Hao J, Zhang S, Zhou Y, Hu X, Shao C. MicroRNA 483-3p suppresses the expression of DPC4/Smad4 in pancreatic cancer. FEBS Lett 2011; 585: 207-213.

16. Ozata DM, Caramuta S, Velazquez-Fernandez D, Akcakaya P, Xie H, Hoog A et al. The role of microRNA deregulation in the pathogenesis of adrenocortical carcinoma. Endoc Relat Cancer 2011; 18: 643-655.

17. Veronese A, Lupini L, Consiglio J, Visone R, Ferracin M, Fornari $F$ et al. Oncogenic role of miR-483-3p at the IGF2/483 locus. Cancer Res 2010; 70: 3140-3149.

18. Veronese A, Visone R, Consiglio J, Acunzo M, Lupini L, Kim T et al. Mutated beta-catenin evades a microRNA-dependent regulatory loop. Proc Natl Acad Sci U S A 2011; 108: 4840-4845.

19. Turchi L, Chassot AA, Rezzonico R, Yeow K, Loubat A, Ferrua B et al. Dynamic characterization of the molecular events during in vitro epidermal wound healing. $J$ Invest Dermatol 2002; 119: 56-63.

20. Chassot AA, Lossaint G, Turchi L, Meneguzzi G, Fisher D, Ponzio G et al Confluenceinduced cell cycle exit involves pre-mitotic CDK inhibition by p27(Kip1) and cyclin D1 downregulation. Cell Cycle 2008; 7: 2038-2046.

21. Kitagawa M, Higashi $\mathrm{H}$, Jung HK, Suzuki-Takahashi I, Ikeda M, Tamai K et al. The consensus motif for phosphorylation by cyclin D1-Cdk4 is different from that for phosphorylation by cyclin A/E-Cdk2. EMBO J 1996; 15: 7060-7069.

22. Takaki T, Fukasawa K, Suzuki-Takahashi I, Semba K, Kitagawa M, Taya $Y$ et al. Preferences for phosphorylation sites in the retinoblastoma protein of D-type cyclin dependent kinases, Cdk4 and Cdk6, in vitro. J Biochem 2005; 137: 381-386.

23. Mazars A, Fernandez-Vidal A, Mondesert O, Lorenzo C, Prevost G, Ducommun B et al. A caspase-dependent cleavage of CDC25A generates an active fragment activating cyclindependent kinase 2 during apoptosis. Cell Death Differ 2009; 16: 208-218.

24. Choi WY, Giraldez AJ, Schier AF. Target protectors reveal dampening and balancing of Nodal agonist and antagonist by miR-430. Science 2007; 318: 271-274.

25. lavarone A, Massague J. Repression of the CDK activator Cdc25A and cell-cycle arrest by cytokine TGF-beta in cells lacking the CDK inhibitor p15. Nature 1997; 387: 417-422.

26. Jinno S, Hung SC, Okayama H. Cell cycle start from quiescence controlled by tyrosine phosphorylation of Cdk4. Oncogene 1999; 18: 565-571.

27. Martin NG, McAndrew PC, Eve PD, Garrett MD. Phosphorylation of cyclin dependen kinase 4 on tyrosine 17 is mediated by Src family kinases. FEBS J 2008; 275: 3099-3109.

28. Boutros R, Dozier C, Ducommun B. The when and wheres of CDC25 phosphatases. Curr Opin Cell Biol 2006; 18: 185-191.

29. Ray D, Kiyokawa H. CDC25A levels determine the balance of proliferation and checkpoint response. Cell Cycle 2007; 6: 3039-3042.

30. Boutros R, Lobjois V, Ducommun B. CDC25 phosphatases in cancer cells: key players? Good targets? Nat Rev Cancer 2007; 7: 495-507.

31. Fernandez-Vidal A, Mazars A, Manenti S. CDC25A: a rebel within the CDC25 phosphatases family? Anticancer Agents Med Chem 2008; 8: 825-831.

32. Zimmerman WC, Erikson RL. Polo-like kinase 3 is required for entry into $S$ phase. Proc Nat Acad Sci USA 2007; 104: 1847-1852.
33. Endicott JA, Noble ME, Tucker JA. Cyclin-dependent kinases: inhibition and substrate recognition. Curr Opin Struct Biol 1999; 9: 738-744.

34. Bartova I, Otyepka M, Kriz Z, Koca J. Activation and inhibition of cyclin-dependent kinase-2 by phosphorylation; a molecular dynamics study reveals the functional importance of the glycine-rich loop. Protein sci 2004; 13: 1449-1457

35. Welburn JP, Tucker JA, Johnson T, Lindert L, Morgan M, Willis A et al. How tyrosine 15 phosphorylation inhibits the activity of cyclin-dependent kinase 2-cyclin A. J Biol Chem 2007; 282: 3173-3181.

36. Stepanova L, Leng X, Parker SB, Harper JW. Mammalian p50Cdc37 is a protein kinasetargeting subunit of Hsp90 that binds and stabilizes Cdk4. Genes Dev 1996; 10: 1491-1502.

37. Matsushime H, Quelle DE, Shurtleff SA, Shibuya M, Sherr CJ, Kato JY. D-type cyclindependent kinase activity in mammalian cells. Mol Cell Biol 1994; 14: 2066-2076.

38. Paternot S, Bockstaele L, Bisteau X, Kooken H, Coulonval K, Roger PP. Rb inactivation in cell cycle and cancer: the puzzle of highly regulated activating phosphorylation of CDK4 versus constitutively active CDK-activating kinase. Cell Cycle 2010; 9: 689-699.

39. Zhao Q, Boschelli F, Caplan AJ, Arndt KT. Identification of a conserved sequence motif that promotes Cdc37 and cyclin D1 binding to Cdk4. J Biol Chem 2004; 279: 12560-12564.

40. Sonkoly E, Wei T, Janson PC, Saaf A, Lundeberg L, Tengvall-Linder M et al. MicroRNAs: novel regulators involved in the pathogenesis of psoriasis? PLOS ONE 2007; 2: e610.

41. Joyce CE, Zhou X, Xia J, Ryan C, Thrash B, Menter A et al. Deep sequencing of small RNAs from human skin reveals major alterations in the psoriasis miRNAome. Hum $\mathrm{Mol}$ Genet 2011; 20: 4025-4040.

42. Kong D, Piao YS, Yamashita S, Oshima H, Oguma K, Fushida S et al. Inflammationinduced repression of tumor suppressor miR-7 in gastric tumor cells. Oncogene 2012; 31: 3949-3960.

43. Wang W, Zhao LJ, Tan YX, Ren H, Qi ZT. MiR-138 induces cell cycle arrest by targeting cyclin D3 in hepatocellular carcinoma. Carcinogenesis 2012; 33: 1113-1120.

44. Wang W, Zhao LJ, Tan YX, Ren H, Qi ZT. Identification of deregulated miRNAs and their targets in hepatitis B virus-associated hepatocellular carcinoma. World J Gastroenterol 2012; 18: 5442-5453.

45. Yi C, Wang Q, Wang L, Huang Y, Li L, Liu L et al. MiR-663, a microRNA targeting p21(WAF1/CIP1), promotes the proliferation and tumorigenesis of nasopharyngeal carcinoma. Oncogene 2012; 31: 4421-4433.

46. Olson P, Lu J, Zhang H, Shai A, Chun MG, Wang Y et al. MicroRNA dynamics in the stages of tumorigenesis correlate with hallmark capabilities of cancer. Genes Dev 2009; 23: 2152-2165.

47. Guled M, Lahti L, Lindholm PM, Salmenkivi K, Bagwan I, Nicholson AG et al. CDKN2A, NF2, and JUN are dysregulated among other genes by miRNAs in malignant mesothelioma -A miRNA microarray analysis. Genes Chromosomes Cancer 2009; 48: 615-623.

48. Doghman M, Wakil AE, Cardinaud B, Thomas E, Wang J, Zhao W et al. Regulation of insulin-like growth factor-mammalian target of rapamycin signaling by microRNA in childhood adrenocortical tumors. Cancer Res 2010; 70: 4666-4675.

49. Dulic V, Stein GH, Far DF, Reed SI. Nuclear accumulation of p21Cip1 at the onset of mitosis: a role at the G2/M-phase transition. Mol Cell Biol 1998; 18: 546-557.

Supplementary Information accompanies the paper on Cell Death and Differentiation website (http://www.nature.com/cdd) 